\title{
Regulation of Neutrophil Extracellular Trap Formation by Anti-Inflammatory Drugs
}

\author{
María José Lapponi, Agostina Carestia, Verónica Inés Landoni, Leonardo Rivadeneyra, \\ Julia Etulain, Soledad Negrotto, Roberto Gabriel Pozner, and Mirta Schattner
}

Laboratory of Experimental Thrombosis (M.J.L., A.C., L.R., J.E., S.N., R.G.P., M.S.) and Laboratory of Physiology of Inflammatory Processes (V.I.L.), Institute of Experimental Medicine (IMEX), CONICET-National Academy of Medicine, Buenos Aires, Argentina

Received January 9, 2013; accepted March 26, 2013

\begin{abstract}
The formation of neutrophil extracellular traps (NETs) is a newly described phenomenon that increases the bacteria-killing ability and the inflammatory response of neutrophils. Because NET generation occurs in an inflammatory microenvironment, we examined its regulation by anti-inflammatory drugs. Treatment of neutrophils with dexamethasone had no effect, but acetylsalicylic acid (ASA) treatment prevented NET formation. NETosis was also abrogated by the presence of BAY 11-7082 $[(E)-3-$ [4-methylphenylsulfonyl]-2-propenenitrile] and Ro 106-9920 [6-(phenylsulfinyl)tetrazolo[1,5-b]pyridazine], two structurally unrelated nuclear factor $-\kappa \mathrm{B}(\mathrm{NF}-\kappa \mathrm{B})$ inhibitors. The decrease in NET formation mediated by ASA, BAY-11-7082, and Ro 106-9920 was correlated with a significant reduction in the
\end{abstract}

phosphorylation of NF- $\kappa \mathrm{B}$ p65 subunit, indicating that the activation of this transcription factor is a relevant signaling pathway involved in the generation of DNA traps. The inhibitory effect of these drugs was also observed when NET generation was induced under acidic or hyperthermic conditions, two stress signals of the inflammatory microenvironment. In a mouse peritonitis model, while pretreatment of animals with ASA or BAY 11-7082 resulted in a marked suppression of NET formation along with increased bacteremia, dexamethasone had no effect. Our results show that NETs have an important role in the local control of infection and that ASA and NF- $\kappa$ B blockade could be useful therapies to avoid undesired effect of persistent neutrophil activation.

\section{Introduction}

Neutrophils are the most abundant leukocytes in human blood and have an essential role in innate immunity as they are the first cells recruited to sites of infection and inflammation (Nathan, 2006). Neutrophils function to engulf microorganisms or opsonized particles and degrade them intracellularly as well as release microbicidal proteins and reactive oxygen species (Segal, 2005). Recently, a novel pathogenkilling mechanism (NETosis) has been described in these cells that involves the extracellular release of microbicidal proteins and their nuclear DNA upon activation by bacterial or fungal species (Brinkmann et al., 2004; Urban et al., 2006). These DNA structures named neutrophil extracellular traps (NETs) are composed of chromatin and are associated mainly with histones; many granular antimicrobial proteins, including elastase, myeloperoxidase, calprotectin, lactoferrin, and metalloprotease 9 , as well as some cytoplasmic proteins that have potent antimicrobial effects are released (Brinkmann et al., 2004; Urban et al., 2009). Thus, NETs bind, disarm, and

This work was supported by the National Agency of Scientific and Technological Support [Grants PICT 2008-0230] and PICT 2011-0733]. dx.doi.org/10.1124/jpet.112.202879. kill microbes extracellularly and independent of phagocytic uptake.

In addition to their antimicrobial properties, NETs may serve as a physical barrier that prevents further spread of the pathogens. Furthermore, retaining granular proteins within the NETs may prevent potentially injurious proteins such as proteases from diffusing away and inducing damage in tissue adjacent to the site of inflammation (Brinkmann and Zychlinsky, 2007). Although it was originally proposed that NETs are formed exclusively in tissues at sites of bacterial or yeast infection, NETs have also been found within blood vessels (specifically in the lung capillaries and liver sinusoids) where they ensnare bacteria in circulation during sepsis (Clark et al., 2007).

In spite of the beneficial effects of this process in pathogen control, the removal of NETs must be regulated to ensure tissue homeostasis, as a high amount of NETs may contribute to collateral damage within inflamed tissues. Excessive NET generation as a result of noninfectious inflammatory processes as well as a lack of DNAses to degrade them is associated with the pathogenesis of inflammatory and autoimmune diseases, including preeclampsia (Gupta et al., 2005), cystic fibrosis (Manzenreiter et al., 2012), and lupus (Hakkim et al., 2010). Moreover, the observation that NETs act as a scaffold for

ABBREVIATIONS: ASA, acetylsalicylic acid; BAY 11-7082, (E)-3-[4-methylphenylsulfonyl]-2-propenenitrile; CFU, colony-forming unit; DMSO, dimethyl sulfoxide; ELISA, enzyme-linked immunosorbent assay; ERK, extracellular signal-regulated kinase; HRP, horseradish peroxidase; MNase, micrococcal nuclease; NET, neutrophil extracellular trap; NF- $\kappa$ B, nuclear factor- $\kappa$ B; PI, propidium iodide; PMA, phorbol 12-myristate 13-acetate; PMN, polymorphonuclear leukocyte; Ro 106-9920, 6-(phenylsulfinyl)tetrazolo[1,5-b]pyridazine; TNF- $\alpha$, tumor necrosis factor $\alpha$. 
thrombus formation (Fuchs et al., 2010, 2012; von Bruhl et al., 2012 ) is increasingly being recognized as a critical phenomenon linking inflammation with venous thrombosis in both infectious and noninfectious clinical settings. NETs are therefore similar to a double-edged sword, functioning not only as an effective antimicrobial first-line defense mechanism but might also leading to organ failure and death if the process is uncontrolled.

Because an inflammatory microenvironment is an essential component of NET formation, we analyzed the effect of current anti-inflammatory drugs including dexamethasone and acetylsalicylic acid (ASA) on NET formation. We found that while dexamethasone had no effect, ASA significantly inhibited NET generation. In addition, two structurally unrelated nuclear factor $-\kappa \mathrm{B}(\mathrm{NF}-\kappa \mathrm{B})$ inhibitors also suppressed NET generation, demonstrating the involvement of the transcription factor NF- $\kappa \mathrm{B}$ in this process and the potential use of these drugs to control NETosis.

\section{Materials and Methods}

Reagents. Phorbol 12-myristate 13-acetate (PMA), propidium iodide (PI), Evans blue dye, ASA, and dexamethasone were purchased from Sigma Aldrich (St. Louis, MO). CELLSTAR culture microplates were purchased at Greiner Bio-One (Monroe, NC). Micrococcal nuclease (MNase) and the cell death detection ELISA ${ }^{\text {PLUS }}$ kit were purchased from Roche Diagnostics (Mannheim, Germany). FicollHypaque (1.077 g/m density) and nitrocellulose membranes from GE Healthcare (Buckinghamshire, UK) and RPMI 1640 medium from Invitrogen (Carlsbad, CA) were used. The NF- $\mathrm{B}$ inhibitors (E)-3-[4methylphenylsulfonyl]-2-propenenitrile (BAY 11-7082) and 6(phenylsulfinyl)tetrazolo[1,5-b]pyridazine (Ro 106-9920) were obtained from Biomol (Plymouth Meeting, PA) and Tocris (Ellisville, MO), respectively. Both drugs were dissolved in dimethyl sulfoxide (DMSO). Tumor necrosis factor $\alpha(\mathrm{TNF}-\alpha)$ was purchased from Peprotech (Veracruz, Mexico). Rabbit anti-neutrophil elastase was obtained from Calbiochem-Merk Millipore (Darmstad, Germany), and anti-rabbit Alexa 488 was obtained from Invitrogen Molecular Probes (Eugene, OR). MacConkey agar was purchased from Britania (Buenos Aires, Argentina). Radioimmunoprecipitation assay lysis buffer and bicinchoninic acid protein assay were purchased from Pierce Chemical (Rockford, IL). Rabbit polyclonal anti-p-NF- $\kappa$ B p65 (Ser311), mouse monoclonal anti-p-extracellular signal-regulated kinase (ERK) (Tyr204), and goat anti-rabbit horseradish peroxidase (HRP) secondary antibody were purchased from Santa Cruz Biotechnology (Dallas, TX). Goat antimouse HRP was obtained from Dako (Glostrup, Denmark). Mouse antiactin antibody was obtained from BD Biosciences (San Jose, CA).

Isolation of Human Neutrophils. Neutrophils were isolated from peripheral blood drawn from healthy donors by Ficoll Hypaque gradient centrifugation and dextran sedimentation, as described previously elsewhere (Negrotto et al., 2006). This study was conducted according to the principles expressed in the Declaration of Helsinki. All patients provided written informed consent for the collection of samples and subsequent analysis. Cell suspensions contained $>96 \%$ neutrophils, as determined by May GrunwaldGiemsa stained cytopreps, and the levels of monocyte contamination were always $<0.2 \%$, as evaluated by CD14 staining and flow cytometry. The cells $\left(4 \times 10^{5} / \mathrm{ml}\right)$ were resuspended in RPMI 1640 medium supplemented with bovine serum albumin (2\%).

NET Formation Assay. Neutrophils $\left(2 \times 10^{5}\right)$ were seeded in 24-well flat-bottom-plates with coverslips, stimulated with PMA or TNF- $\alpha$ at $\mathrm{pH} 7.4$, and placed in a humidified incubator at $37^{\circ} \mathrm{C}$ with $\mathrm{CO}_{2}(5 \%)$ for 180 minutes. For quantification purposes, NETs were then digested with $\mathrm{MNase}(500 \mathrm{mU} / \mathrm{ml})$ for 10 minutes at $37^{\circ} \mathrm{C}$.
To study NET formation at hyperthermic conditions, neutrophils were placed in three different incubators with temperatures of $37^{\circ}$, $40^{\circ}$, and $42^{\circ} \mathrm{C}$.

To induce acidosis, the $\mathrm{pH}$ of the culture medium was adjusted to $7.4,7.0$, or 6.5 by the addition of isotonic $\mathrm{HCl}(1 \mathrm{~N})$ solution before the stimulation with PMA, as described earlier. Neutralization before treatment with MNase was achieved by adding a precalculated volume of isotonic $\mathrm{NaOH}(1 \mathrm{~N})$ solution.

In selected experiments, neutrophils were first incubated for 30 minutes with ASA, dexamethasone, BAY 11-7082, Ro 106-9920, or vehicle (controls). ASA and dexamethasone were dissolved in RPMI. Stock solutions of BAY 11-7082 and Ro 106-9920 were prepared in DMSO and were further diluted in RPMI 1640 medium. The final DMSO concentration $(0.1 \% \mathrm{v} / \mathrm{v})$ did not have a toxic effect. All drugs were freshly prepared for each experiment.

Immunofluorescence Assays. After PMA or TNF- $\alpha$ stimulation, cells were fixed with PFA (4\%), permeabilized with triton $(0.25 \%)$, stained with PI $(2 \mu \mathrm{g} / \mathrm{ml})$ washed and mounted on slides with PolyMount. In selected experiments, the generation of NETs was confirmed by labeling the cells with PI and antibody against elastase. Images for NET evaluation were taken and analyzed by confocal fluorescence microscopy, using an Olympus FV-1000 (Tokyo, Japan) equipped with a Plapon $60 \times / \mathrm{NA1} 42$ objective. In vivo, the NET contribution to the bacterial count in the blood was determined by i.p. inoculation of MNase (100 U/mouse or $1 \mathrm{mg} / \mathrm{kg}$, prepared in RPMI 1640).

Quantification of Extracellular DNA. DNA released from neutrophils during NET formation was digested with MNAse (500 $\mathrm{mU} / \mathrm{ml})$. EDTA (5 mM) was added to stop nuclease activity; supernatants were collected and stored at $-20^{\circ} \mathrm{C}$ until assayed. Nucleosomal DNA or complexes of DNA bound to histones were measured in the supernatants using commercially available cell death detection ELISA $^{\text {PLUS }}$ kit according to the manufacturer's instructions. This test is a sandwich enzyme-linked immunosorbent assay (ELISA) using two monoclonal antibodies against histones and DNA that specifically detects mono- and oligo-nucleosomes derived from eukaryotic cells as a result of the MNase treatment of NET. The calibration curve was constructed using a standard of nucleosomal DNA of a known concentration.

Quantitation of Apoptosis by Fluorescence Microscopy. Neutrophils were treated with or without BAY 11-7082 (2.5 $\mu \mathrm{M})$, Ro 106-9920 $(2.5 \mu \mathrm{M})$, or ASA $(5 \mathrm{mM})$ for 180 minutes and washed; apoptosis was evaluated 17 hours later by fluorescence microscopy. Cells were labeled with a mixture of the fluorescent DNA-binding dyes acridine orange $(100 \mu \mathrm{g} / \mathrm{ml})$ to determine the percentage of cells that had undergone apoptosis and ethidium bromide $(100 \mu \mathrm{g} / \mathrm{ml})$ to differentiate between viable and nonviable cells (Negrotto et al., 2006). At least 300 cells were scored in each experiment.

Immunoblotting. Neutrophils were preincubated or not with the anti-inflammatory drugs and stimulated for 40 minutes with PMA (50 $\mathrm{nM})$. Lysates were prepared using radioimmunoprecipitation assay lysis buffer with a protease inhibitor cocktail. Cell debris was separated by centrifugation, and protein in the supernatant was quantified using the bicinchoninic acid protein assay. Samples were then frozen at $-80^{\circ} \mathrm{C}$ until use. Equal amounts of proteins $(40 \mu \mathrm{g} /$ sample) were separated by electrophoresis (12\% SDS-polyacrylamide gels). Proteins were then electrotransferred onto nitrocellulose membranes. After blocking, membranes were incubated overnight at $4^{\circ} \mathrm{C}$ with a rabbit polyclonal anti-p-N- $\kappa$ B p65 (Ser311), mouse monoclonal anti-p-ERK 1/2 (Tyr204), and mouse monoclonal antiactin antibody followed by a goat anti-rabbit HRP or anti-mouse HRP secondary antibody. Protein bands were visualized by enhanced chemiluminescence. Immunoblotting results were quantified using Gel-Pro Analyzer 3.1 software, and the values from blot reprobes were used for monitoring equal protein loads.

Mice Studies. BALB/c mice were bred in the animal facility of the Institute of Experimental Medicine. The mice studies were conducted according to principles set forth in the guide for the care and use of laboratory animals (Office of Laboratory Animal Welfare, 1985; 
http://grants.nih.gov/grants/olaw/references/phspol.htm). Female mice aged 9-16 weeks and weighing 20-25 g were used throughout the experiments. They were maintained under a 12-hour light/dark cycle at $22^{\circ} \pm 2^{\circ} \mathrm{C}$ and fed with a standard diet and water ad libitum.

Polymicrobial Challenge. The cecum of an untreated mouse was removed, and the intestinal content was left at $37^{\circ} \mathrm{C}$ for 24 hours in sterile saline solution supplemented with $10 \%$ fetal calf serum in aerobic conditions. Routine bacteriologic identification revealed that the bacteria present in the inoculum belonged to Enterococcus and Streptococcus spp (Landoni et al., 2012). Dosage measurements of the bacteria were performed in agar MacConkey plates. Aerobic enriched bacteria $\left(5 \times 10^{7}\right)$ were injected i.p., and 3 hours later the peritoneal lavage was performed with sterile saline solution as described below. In selected experiments, mice were injected i.p. with one dose $(60$ minutes) of ASA (100 mg/kg, dissolved in RPMI 1640) or dexamethasone $(2.5 \mathrm{mg} / \mathrm{kg}$, dissolved in phosphate-buffered saline) before the polymicrobial challenge; two doses of BAY 11-708 [15 minutes before and 90 minutes after, $5 \mathrm{mg} / \mathrm{kg}$, dissolved in saline solution/DMSO (25\%)]; or MNase (15 minutes before injection with bacteria, $1 \mathrm{mg} / \mathrm{kg}$, dissolved in RPMI 1640).

Peritoneal Lavage. Animals were euthanized, and the peritoneal content was collected by peritoneal lavage, as previously described elsewhere (Negrotto et al., 2006). Briefly, the skin of the abdomen was cut open at the midline after thorough disinfection and without injuring the muscle. Sterile saline solution $(2 \mathrm{ml})$ was injected into and aspirated out of the peritoneal cavity twice, using a sterile syringe and needle, to rinse out the content from the peritoneal cavity. The number of neutrophils was determined microscopically after staining with Turk's solution, and the peritoneal liquid was used to determine the bacterial clearance, as described below.

Bacterial Cultures. Blood and peritoneal lavage fluid were obtained; the cells were lysed with sterile distilled water. Aliquots of serial log dilutions of peritoneal fluid and blood were plated onto MacConkey agar, which is a selective growth medium for intestinal bacteria. Plates were grown under aerobic conditions at $37^{\circ} \mathrm{C}$, and colony-forming units (CFU) were counted after an overnight incubation. Bacterial counts are expressed as CFU per milliliter of peritoneal lavage fluid or blood.

In Vivo Evaluation of NET Formation. In vivo evaluation of NET formation was performed using a mouse model of peritonitis, as previously described elsewhere (Landoni et al., 2012). NETs were induced with i.p. bacterial injection, and after 3 hours the peritoneal cells were carefully collected and gently seeded on coverslides. Immunostaining was performed as described above.

Vascular Permeability Assay. Evans blue dye $(30 \mathrm{mg} / \mathrm{kg}$ in phosphate-buffered saline) was injected i.v., and 20 minutes later, the mice were euthanized, and peritoneal lavage was performed with $1 \mathrm{ml}$ of sterile saline solution. The Evans blue dye collected from the peritoneal cavity was measured spectrophotometrically at $560 \mathrm{~nm}$ (Kanaoka et al., 2001).

Statistical Analysis. Data are expressed as mean \pm S.E.M. The analysis of variance plus the Newman-Keuls or Dunnet's (for mice studies) test were employed to determine statistically significant differences between the groups. $P<0.05$ was considered statistically significant.

\section{Results}

Effects of Anti-Inflammatory Drugs on NET Formation. In the first experiments, we determined the formation of NETs by stimulation of neutrophils with PMA or the proinflammatory cytokine TNF- $\alpha$. Double labeling of the cells with PI to identify DNA and with an antibody against elastase, a neutrophil granular-derived protein that decorates the DNA strands, demonstrated that both stimuli triggered the formation of NETs (Fig. 1). Albeit NETs are usually generated during inflammatory clinical settings, how anti- inflammatory drugs affect this process is not completely understood at present. Hence, we analyzed the effects of the most commonly used anti-inflammatory drugs, ASA and dexamethasone, on NET formation triggered by PMA or TNF- $\alpha$. We found that while PMA-induced NET formation was not modified by ASA ( $1 \mathrm{mM})$, it was markedly inhibited by higher ASA concentrations ( $5 \mathrm{mM}$ ) (Fig. 2, A and B). The amount of NETs was similar in controls and in dexamethasonetreated neutrophils. To further corroborate the fluorescence microscopy studies, NET formation was measured by ELISA. The quantification of the released DNA confirmed that the formation of extracellular traps was significantly diminished by ASA (Fig. 2B). The effect of both ASA and dexamethasone was similar when NETs were triggered by stimulation of neutrophils with TNF- $\alpha$ (Fig. 2, A and B).

The transcription factor $\mathrm{NF}-\kappa \mathrm{B}$ is a key regulator of inflammation and therefore plays a pivotal role in a wide range of inflammatory diseases (Manzenreiter et al., 2012). Because the effects of high ASA concentrations are not only related to cyclo-oxygenase acetylation and include inhibition of NF- $\kappa$ B activation (Yin et al., 1998), we next investigated the role of NF- $\kappa$ B blockade on NET formation. Neutrophils were incubated with BAY 11-7082 or Ro 106-9920, two structurally unrelated specific inhibitors of $\mathrm{I} \kappa \mathrm{B} \alpha$ (inhibitor of $\kappa \mathrm{B}$ ) phosphorylation (Pierce et al., 1997) and $\mathrm{I} \kappa \mathrm{B} \alpha$ ubiquitination (Swinney et al., 2002), respectively. Figure 2, A and B, shows that the release of DNA traps was markedly impaired by these drugs. Western blot analysis confirmed that phosphorylation of NF- $\kappa \mathrm{B}$ p65 subunit triggered by PMA was significantly inhibited by pretreatment of the neutrophils with BAY 11-7082 or Ro 106-9920 in a concentration-dependent manner (Fig. 2C). In addition they showed that while $1 \mathrm{mM}$ of ASA did not modify activation of NF- $\kappa \mathrm{B}$, the phosphorylation of p65 subunit of NF- $\kappa \mathrm{B}$ was markedly impaired in polymorphonuclear leukocyte (PMN) preincubated with $5 \mathrm{mM}$ of ASA. On the other hand, dexamethasone even at $10 \mu \mathrm{M}$ had no effect. ERK phosphorylation, a pathway recently described to be implicated in NET formation (Fig. 2C) (Hakkim et al., 2011) was not modified by any of the drugs employed.

The absence of nuclear changes determined in morphologic studies indicated that inhibition of NET formation mediated by either ASA or NF- $\kappa$ B inhibitors was not associated to significant drug-induced neutrophil apoptosis and therefore did not account for the observed inhibition (\% apoptosis: Control: $55 \pm$ 3; BAY $2.5 \mu \mathrm{M}$ : $62 \pm 4$; Ro $2.5 \mu \mathrm{M}$ : $64 \pm 4$; ASA $5 \mathrm{mM}$ : $55 \pm 4$ ).

Effect of Anti-Inflammatory Drugs on NET Formation under Acidosis or Hyperthermic Conditions. Because NET generation occurs in an inflammatory microenvironment, we next examined whether the inhibitory effect of the antiinflammatory drugs was still evident when NETs are generated under characteristic stress signals of this milieu such as hyperthermia or acidosis. Interestingly, ELISA studies showed that the increase in temperature from the physiologic $37^{\circ}$ to $42^{\circ} \mathrm{C}$ as well as the decrease in $\mathrm{pH}$ values markedly augmented NET formation (Fig. 3A). Despite this increase, ASA or NF- $\kappa$ B inhibitors were still effective as both exerted a significant suppression of NETosis under these stressful conditions (Fig. 3B).

\section{ASA and BAY 11-7082 Impair In Vivo NET Formation.} Having demonstrated that ASA and the NF- $\kappa$ B inhibitors had profound inhibitory effects on NET formation in vitro, we next explored the effects of these drugs in an in vivo model of peritonitis (Landoni et al., 2012). Consistent with our in vitro 
PI
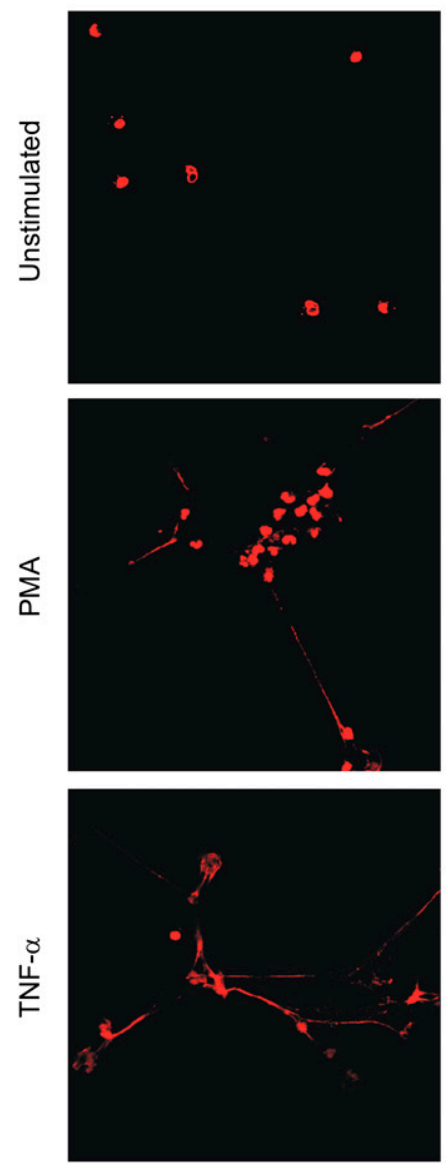

Elastase
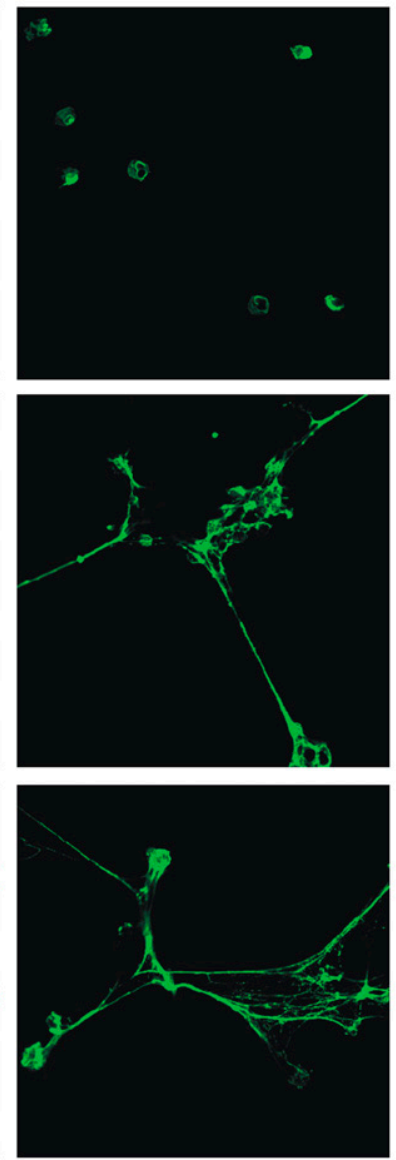

Merge
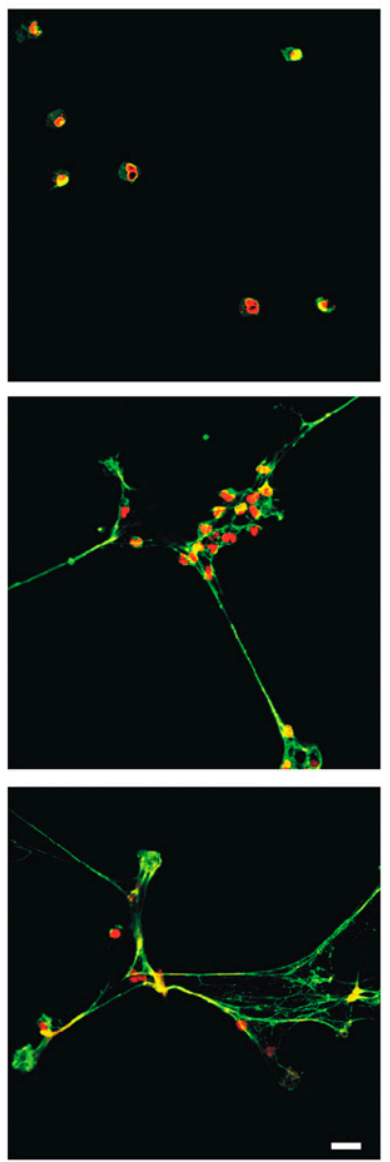

Fig. 1. NET induction by PMA and TNF- $\alpha$. Neutrophils were stimulated or not with PMA $(50 \mathrm{nM})$ or TNF- $\alpha(20 \mathrm{ng} / \mathrm{ml})$ for 180 minutes. Cells were then fixed and stained with PI for DNA (red) and the specific marker antineutrophil elastase (green) and were visualized by confocal fluorescence microscopy $(n=3)$. Original magnification, $60 \times$ (scale bar: $20 \mu \mathrm{m}$ ). results, we found that in mice pretreated with ASA or BAY 11-7082, NET formation was significantly inhibited in the peritoneal cavity and also that PMN migration toward the infection site was decreased (Fig. 4, A and B). To further elucidate the role of NETosis in the clearance of bacterial infections, we also quantified the bacteria load in the peritoneal cavity and blood of animals that were treated with ASA, BAY 11-7082, dexamethasone, or not treated. Although peritoneal bacterial counts (expressed as $\mathrm{CFU} / \mathrm{ml}$ ) were significantly lower in ASA-treated mice compared with control animals, the CFUs were similar to mice infused with BAY 11-7082 (Fig. 4C). Interestingly, the treatment of mice with either anti-inflammatory drug before the bacterial challenge, resulted in a marked increase in bacteremia (Fig. 4D), indicating that NETs contribute toward limiting bacterial dissemination. Dexamethasone treatment did not alter PMN migration, NET formation, or bacterial loads in peritoneum or blood, further supporting our in vitro results.

To analyze whether increased bacteremia was due to an augmented vascular permeability mediated by the antiinflammatory drugs, we next evaluated the presence of Evans blue dye in the peritoneal cavity, which indicates dye migration from the vasculature to the tissues. Spectrophotometrical analysis of peritoneal lavage fluid collected from infected and noninfected mice treated with ASA, BAY 117082 , or dexamethasone indicated that infection by itself markedly increased vascular permeability; however, this effect was not further increased by any of the employed anti-inflammatory drugs (Fig. 4E). Additionally, bacteremia was evaluated after MNase treatment, which disrupts NETs but does not alter the number of neutrophils that migrate to the peritoneum. Fig. 4D shows that bacteremia was significantly increased when NETs were disrupted enzymatically. Together, these data indicate that in this experimental model of peritonitis inhibition of NETosis failed to locally restrict the infection.

\section{Discussion}

Recently, NETs were identified as an alternative mechanism of pathogen killing. Although NET formation may have a beneficial effect for the host in terms of isolating and preventing the spread of invading bacteria, the persistent activation of neutrophils or deficient NET degradation by DNAses can also promote injury to the host (Hakkim et al., 2010; Caudrillier et al., 2012). Thus, although there are beneficial effects of this process in pathogen control, NET removal must be regulated to ensure tissue homeostasis. The mechanisms or molecules involved in the regulation of NET formation are not yet completely known. Although still a matter of debate, the release of DNA to the extracellular milieu is considered a cell death program (NETosis) distinct from apoptosis and necrosis (Fuchs et al., 2007). Moreover, it has been recently suggested that the interplay between autophagy, reactive oxygen species formation, and peptidylarginine deiminase 4-dependent histone citrullination promotes the collapse of both nuclear and granular neutrophil membranes and mediates intracellular chromatin decondensation 
A
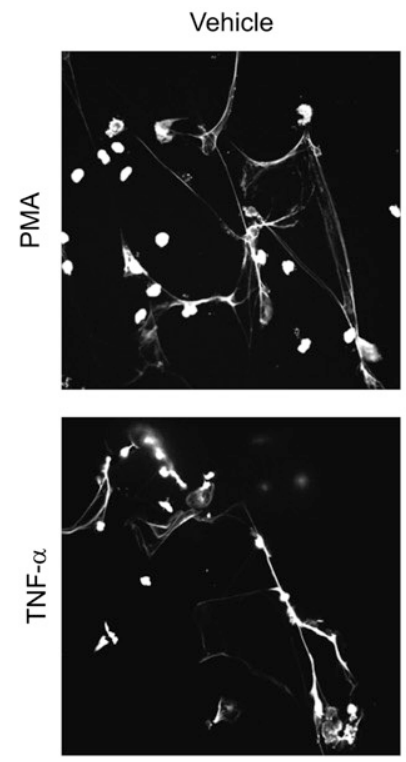

B

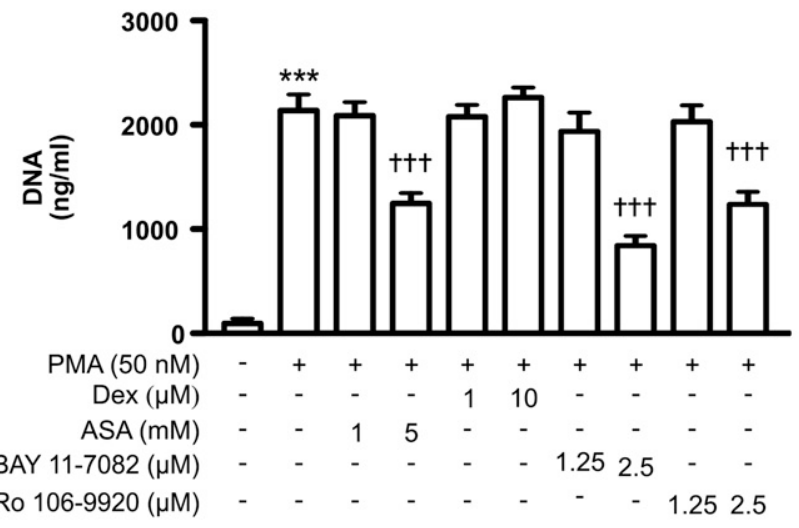

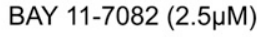
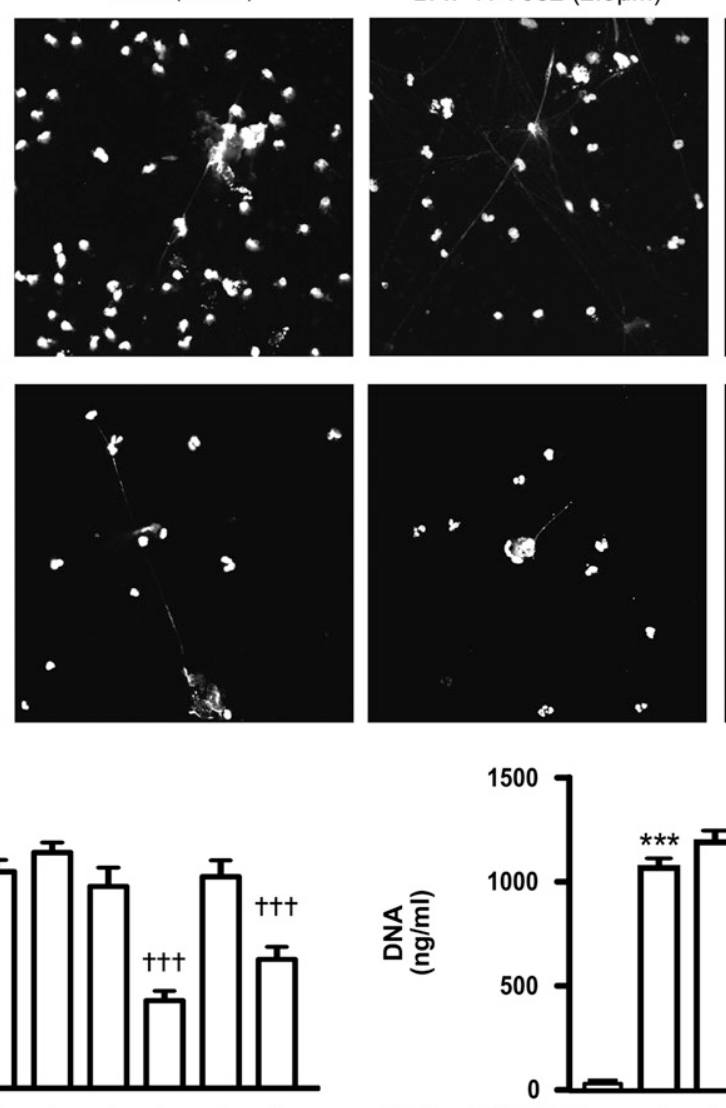

TNF- $\alpha(20 \mathrm{ng} / \mathrm{ml})$

$\operatorname{Dex}(\mu \mathrm{M})$.

$\mathrm{ASA}(\mathrm{mM})$ -

BAY 11-7082 $(\mu \mathrm{M})$ -

Ro 106-9920 ( $\mu \mathrm{M})$ -

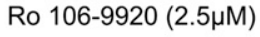
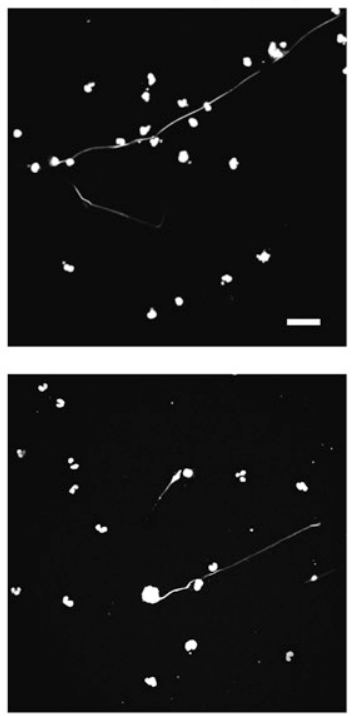

1500

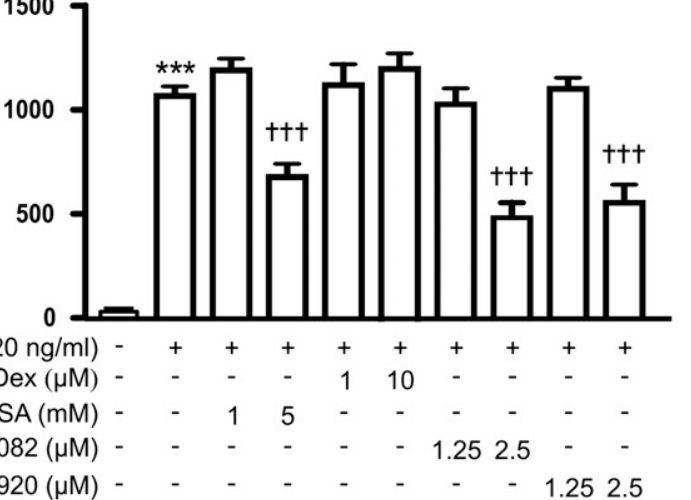

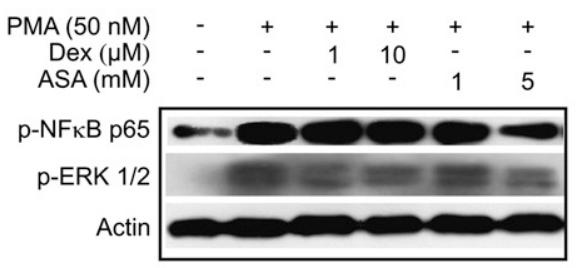

pERK

pERK-1

pERK-2
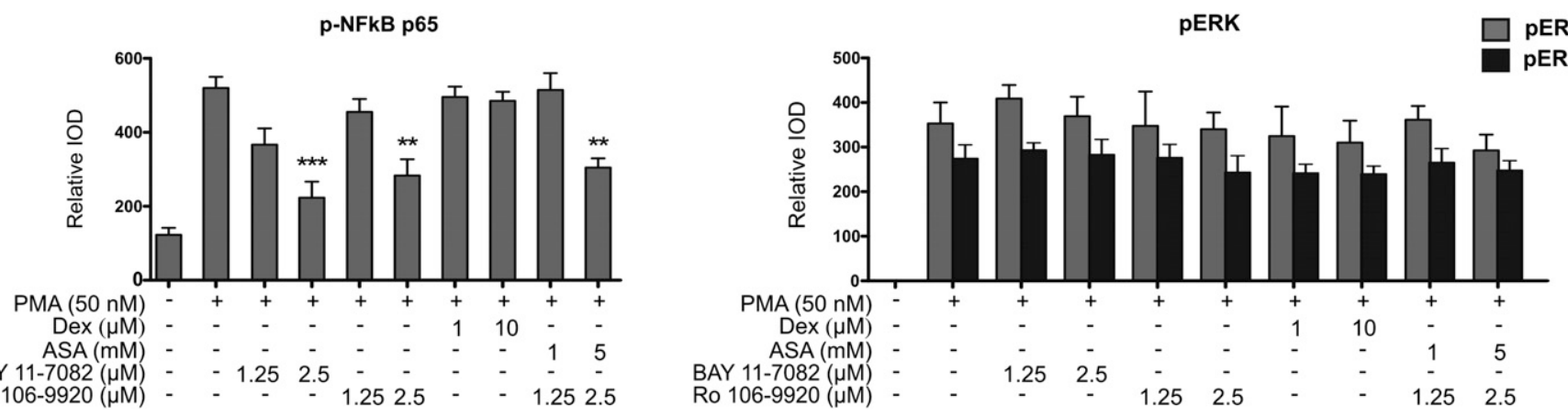
Ro 106-9920 $(\mu \mathrm{M})$

1.252 .5

Fig. 2. Effect of anti-inflammatory drugs on the ability of neutrophils to release NETs. Neutrophils were pretreated or not (vehicle) for 30 minutes with ASA, dexamethasone (Dex), BAY 11-7082, or Ro 106-9920, followed by a 180-minute activation with PMA or TNF- $\alpha$. (A) DNA was stained with PI and visualized by confocal fluorescence microscopy $(n=3$ ). Original magnification, $60 \times$ (scale bar: $20 \mu \mathrm{m}$ ). (B) NET-associated DNA was quantified in the supernatants by ELISA $(n=4$; $* * * P<0.001$ versus unstimulated; $\dagger \dagger \dagger P<0.001$ versus PMA or TNF- $\alpha$-stimulated neutrophils). (C) Western blot analysis of PMA-stimulated neutrophils treated with various concentrations of anti-inflammatory drugs. Briefly, neutrophils were preincubated at $37^{\circ} \mathrm{C}$ for 30 minutes with antiinflammatory drugs, then stimulated for 40 minutes with PMA $(50 \mathrm{nM})$; the lysates were immunoblotted with anti-p-NF- $\kappa \mathrm{B}$ p65, pERK1/2. Each membrane was reprobed with antiactin antibody to calculate the relative IOD using GEL-PRO software $(n=3 ; * * * P<0.001 ; * * P<0.01$ versus PMA-stimulated neutrophils). 
A

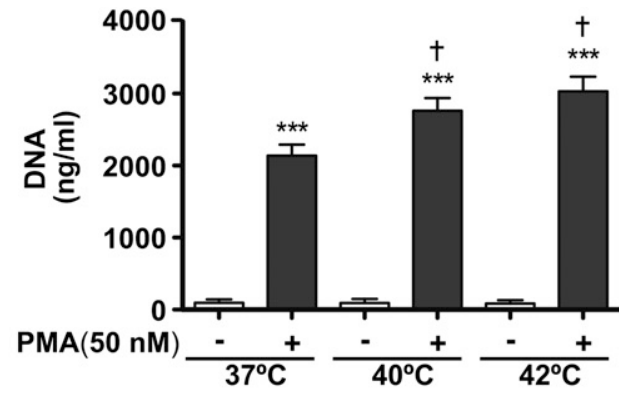

B

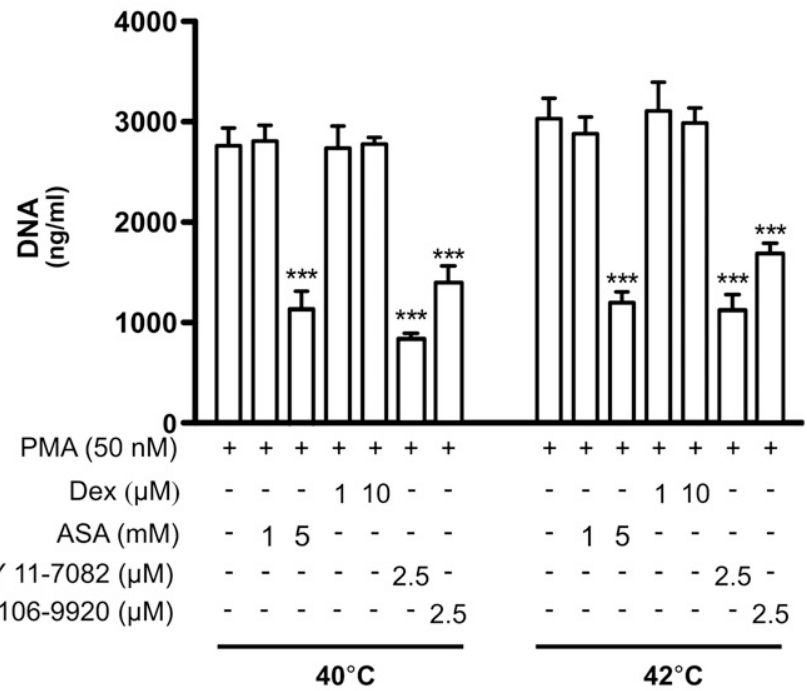

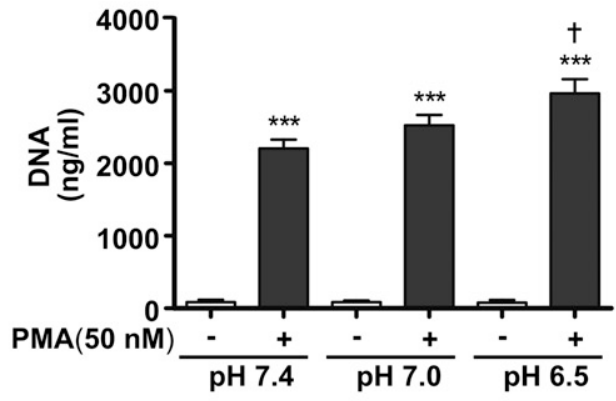

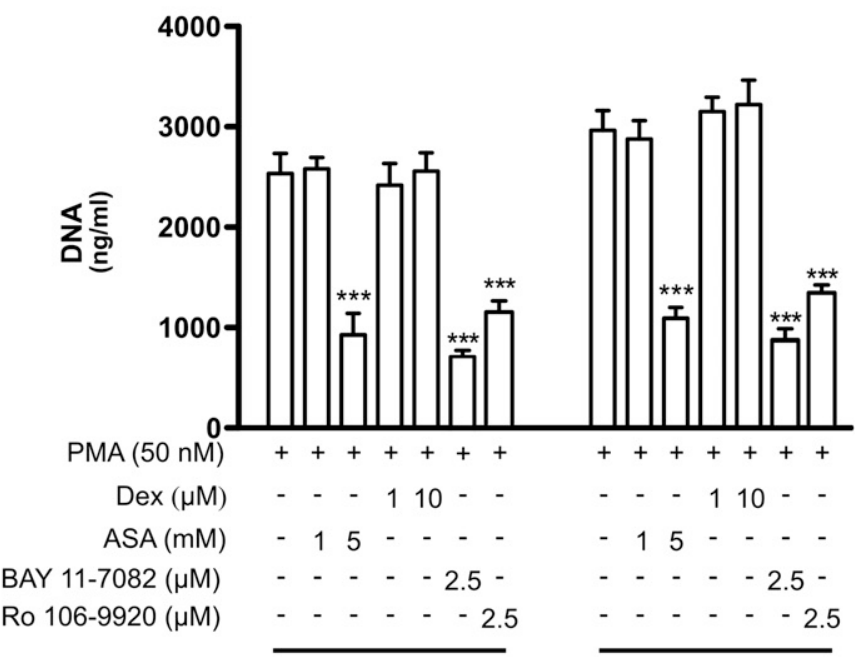

$\mathrm{pH} 7.0$

pH 6.5

Fig. 3. ASA and NF- $\kappa$ B inhibitors also effectively suppressed the increased NETosis under hyperthermic or acidic conditions. (A) Neutrophils were stimulated or not with PMA at different temperatures or $\mathrm{pH}$ values for 180 minutes, and NET-associated DNA was determined by ELISA $(n=4 ; * * * P<$ 0.001 versus unstimulated; $\dagger P<0.05$ versus PMA-stimulated neutrophils at $37^{\circ} \mathrm{C}$ or $\mathrm{pH} 7.4$ ). (B) Neutrophils set at hyperthermic or acidic conditions were pretreated or not (vehicle) for 30 minutes with ASA, dexamethasone, BAY 11-7082, or Ro 106-9920, followed by a 180-minute activation with PMA. NET-associated DNA was quantified in the supernatants by ELISA $(n=4 ; * * P<0.001$ versus PMA-stimulated neutrophils at each condition).

while inhibiting the apoptotic machinery (Remijsen et al., 2011).

Because NETs and inflammation are concomitant events, we explored the effects of the most commonly used antiinflammatory drugs worldwide, ASA ( $5 \mathrm{mM})$ and dexamethasone. We found that, in contrast with dexamethasone, ASA markedly inhibited NET formation. Interestingly, the antiinflammatory effects of the ASA concentration ( $5 \mathrm{mM}$ ) used in this study are generally attributed to its action on cyclooxygenase independent targets, including the inhibition of NF- $\kappa$ B (Yin et al., 1998; Negrotto et al., 2006). Thus, our findings suggested that this major transcription factor involved in the inflammatory response could be a key regulatory factor of NET formation. In fact, the profound inhibition of NETs by two structurally different inhibitors of NF- $\kappa \mathrm{B}$ activation together with previous studies showing that PMA or TNF- $\alpha$ stimulation of neutrophils induces both the nuclear accumulation of NF- $\kappa \mathrm{B} / \mathrm{Rel}$ proteins and the concomitant degradation of cytoplasmic $\mathrm{I} \kappa \mathrm{B}-\alpha$ (McDonald et al., 1997) strongly supports a role of NF- $\kappa \mathrm{B}$ in the biogenesis of NETs. Furthermore, Western blot studies have shown that while treatment of neutrophils with ASA $5 \mathrm{mM}$ or $2.5 \mu \mathrm{M}$ Ro 106-9920 or BAY 11-7082 resulted in a significant impairment of the PMA-induced phosphorylation of NF- $\kappa \mathrm{B}$ p65 subunit, dexamethasone or $1 \mathrm{mM}$ ASA had no effect. In addition, none of the drugs used interfered with the ERK pathway. Altogether, these data strongly indicate that activation of NF- $\kappa \mathrm{B}$ is a signaling pathway involved in the generation of NETs.

Extracellular acidosis, a hallmark of the inflammatory milieu, may intensify acute inflammatory responses by inducing neutrophil activation as well as by delaying spontaneous apoptosis and extending neutrophil functional life span (Trevani et al., 1999). Similar to these findings, our data showing that extracellular acidosis fosters the formation of NETs strongly suggests that low $\mathrm{pH}$ values may contribute to the amplification of neutrophil-mediated inflammatory responses but also promote neutrophil bactericidal responses through the induction of NETosis and the inhibition of apoptosis.

It has been previously shown that acute or long-term concurrent exposure to febrile range hyperthermia exerts multiple actions that profoundly increase neutrophil recruitment, enhance pathogen clearance, and increase lethal lung injury in mouse models of pneumonia and hyperoxia (Hasday et al., 2003). Furthermore, these consequences to innate immune function and regulation of inflammation were also observed after exposure to long-term hyperthermia (Tulapurkar et al., 2011).

Interestingly, because the bacterial proliferation rate appears to not be influenced by high temperatures (Jiang et al., 2000), it was suggested that the reduced pathogen burden could have been achieved through effects on host bacterial clearance mechanisms. Our present findings suggest that an increase of NET generation could be one of the 
ASA

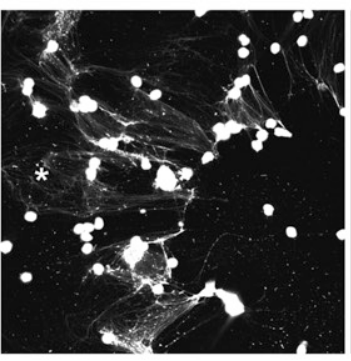

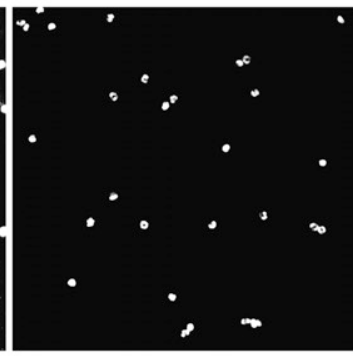

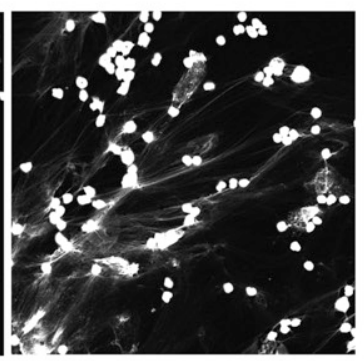

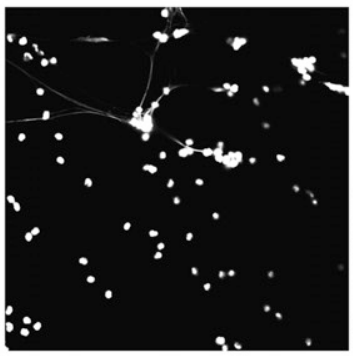

Dex

D

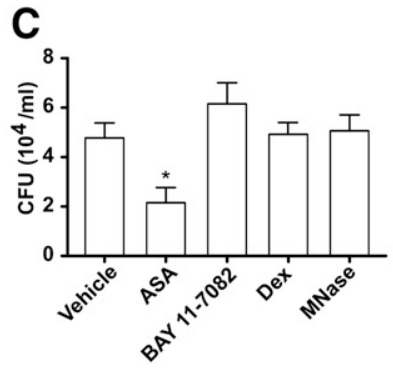

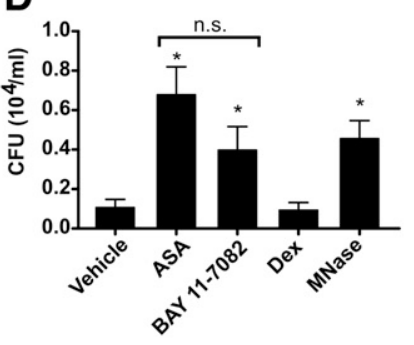

E

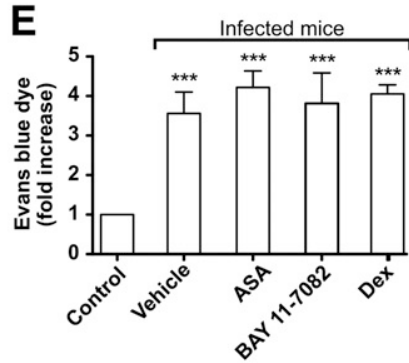

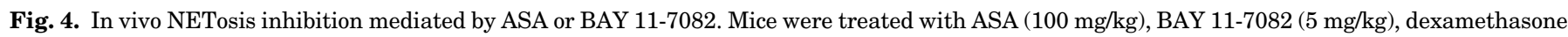

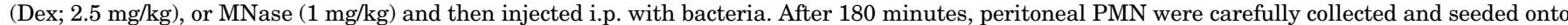

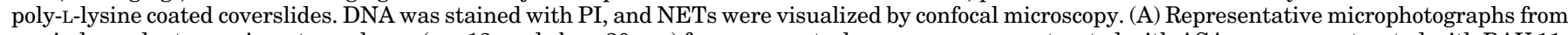

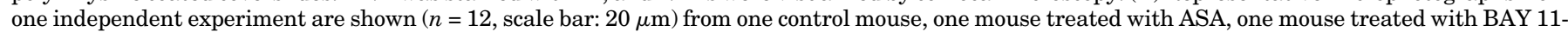

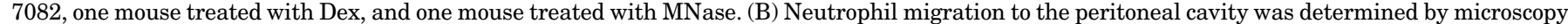

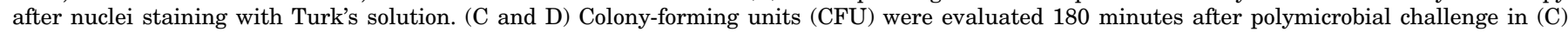

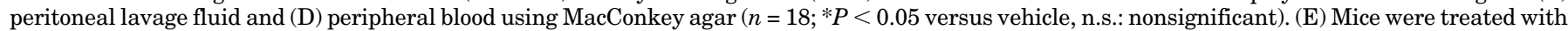

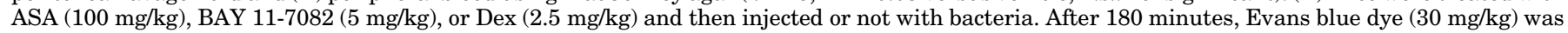

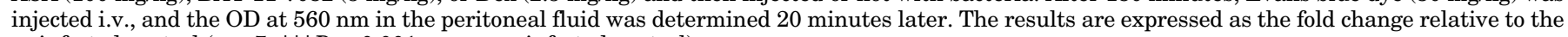
uninfected control ( $n=7 ; * * * P<0.001$ versus uninfected control).

mechanisms responsible for both efficient pathogen clearance and the lung injury observed under experimental hyperthermia conditions.

We found that the increased NET generation triggered by either low $\mathrm{pH}$ values or heat is susceptible to inhibition by ASA or NF- $\kappa$ B inhibitors, suggesting a potential use of these drugs to prevent undesired tissue damage due to an uncontrolled inflammatory response. This hypothesis is further supported by our in vivo experiments showing that pretreating mice with both ASA or BAY 11-7082 but not dexamethasone markedly impaired NET formation in the peritoneum of infected mice.

It was recently reported that ASA ingestion may be beneficial for the treatment of transfusion-related acute lung syndrome (Caudrillier et al., 2012). Interestingly, the effect of ASA in this study was not associated with an interference of ASA with the ability of neutrophils to induce NETs but rather with its inhibitory effect on platelet activation, which is a phenomenon involved in NET formation. Because the doses of ASA used in both studies were the same, our data further suggest that the mechanistic bases for ASA treatment would include not only the suppression of platelet activation but also a blockade of neutrophil NF- $\kappa \mathrm{B}$ activity.

Although the use of aspirin as an anti-inflammatory therapy is widely accepted, the use of NF- $\kappa \mathrm{B}$ inhibitors is just emerging. In this sense, experimental studies in animals showed that alteration of the NF- $\kappa$ B pathway using BAY 117082 yielded beneficial results in functional, sensorimotor, and biochemical deficits associated with diabetic neuropathy (Kumar et al., 2012) as well as a decrease in the risk of myocardial injury associated to ischemia reperfusion (Kim et al., 2010). Furthermore, it has been shown that selective inhibition of NF- $\kappa \mathrm{B}$ activation in inflammatory cells could be a treatment option for patients with inflammatory bowel disease (Dijkstra et al., 2002) and patients with an early stage of ankylosing spondylitis (Chen and Liu, 2006) or rheumatoid arthritis (Mucke, 2012).

We observed that although ASA significantly decreased the number of CFU in peritoneum, BAY 11-7082 showed no effect. The differences between the CFU counts observed between ASA and BAY 11-7082 treatment could be explained by the reported ASA-mediated anti-inflammatory effects not associated with the inhibition of $\mathrm{NF}-\kappa \mathrm{B}$ activation. In this sense, ASA through the aspirin-triggered lipoxins and resolvins promotes phagocytosis-enhancing bacterial clearance (Serhan et al., 2000; Scannell and Maderna, 2006; Prescott and McKay, 2011; Dalli et al., 2013). In contrast, NF- $\kappa$ B inhibition by BAY 11-7082 has been reported to impair phagocytosis (Giraldo et al., 2010). In addition, the observation that during the antimicrobial response neutrophils first kill bacteria by phagocytosis and then by NETs (Fuchs et al., 2007) could contribute to the differences found between ASA and BAY 11-7082 in the bacterial count. Although NETosis suppression appears to be beneficial in some sterile inflammatory diseases, we also found that inhibition of NET formation by ASA and BAY 11-7082 leads to bacterial dissemination toward the bloodstream. This effect was not due to an increase in vascular permeability mediated by the anti-inflammatory drugs; it was also observed in the animals treated with DNase, suggesting that, at least in this mouse model of peritonitis, NET formation helps to localize the infection and prevents bacterial dissemination. 
Increased bacteremia mediated by salicylate treatment was previously reported in experimental peritonitis (Spagnuolo and Ellner, 1979). In that early study, the ability of neutrophils to generate NETs was still unknown. Thus, the increased bacteremia was linked to a decreased influx of peritoneal granulocytes early in the course of infection, which allowed pathogen multiplication. Although extrapolation of mouse data to humans is not direct, the adverse influence of NET inhibition in the outcome of infections should also be considered in the development of new therapies.

Neutrophils are short-lived inflammatory cells that are exposed to cytokines, acidosis, and high temperatures during systemic fever and at sites of local inflammation in several infectious and noninfectious diseases including cancer. Our results indicate that NF- $\kappa \mathrm{B}$ activation is a relevant mediator in the genesis of these extracellular DNA traps, both in vitro and in vivo. Although limiting NET formation appears to be helpful in sterile inflammatory diseases, more research is needed to understand whether this applies to infectious diseases as well.

\section{Acknowledgments}

The authors thank Héctor Costa and Gabriela Camerano for their excellent technical assistance.

\section{Authorship Contributions}

Participated in research design: Schattner, Lapponi.

Conducted experiments: Lapponi, Carestia, Landoni, Rivadeneyra,

Etulain, Negrotto, Pozner.

Contributed new reagents or analytic tools: Landoni.

Performed data analysis: Schattner, Lapponi, Carestia, Pozner.

Wrote or contributed to the writing of the manuscript: Schattner.

\section{References}

Brinkmann V, Reichard U, Goosmann C, Fauler B, Uhlemann Y, Weiss DS, Weinrauch Y, and Zychlinsky A (2004) Neutrophil extracellular traps kill bacteria. Science 303: 1532-1535.

Brinkmann V and Zychlinsky A (2007) Beneficial suicide: why neutrophils die to make NETs. Nat Rev Microbiol 5:577-582.

Caudrillier A, Kessenbrock K, Gilliss BM, Nguyen JX, Marques MB, Monestier M, Toy P, Werb Z, and Looney MR (2012) Platelets induce neutrophil extracellular traps in transfusion-related acute lung injury. J Clin Invest 122:2661-2671.

Clark SR, Ma AC, Tavener SA, McDonald B, Goodarzi Z, Kelly MM, Patel KD, Chakrabarti S, McAvoy E, and Sinclair GD, et al. (2007) Platelet TLR4 activates neutrophil extracellular traps to ensnare bacteria in septic blood. Nat Med 13: $463-469$

Chen J and Liu C (2006) Is sulfasalazine effective in ankylosing spondylitis? A systematic review of randomized controlled trials. J Rheumatol 33:722-731.

Dalli J, Winkler JW, Colas RA, Arnardottir H, Cheng CY, Chiang N, Petasis NA and Serhan CN (2013) Resolvin d3 and aspirin-triggered resolvin d3 are potent immunoresolvents. Chem Biol 20:188-201.

Dijkstra G, Moshage H, and Jansen PL (2002) Blockade of NF- $\kappa$ B activation and donation of nitric oxide: new treatment options in inflammatory bowel disease? Scand J Gastroenterol Suppl (Suppl):37-41.

Fuchs TA, Abed U, Goosmann C, Hurwitz R, Schulze I, Wahn V, Weinrauch Y, Brinkmann V, and Zychlinsky A (2007) Novel cell death program leads to neutrophil extracellular traps. J Cell Biol 176:231-241.

Fuchs TA, Brill A, Duerschmied D, Schatzberg D, Monestier M, Myers DD, Jr, Wrobleski SK, Wakefield TW, Hartwig JH, and Wagner DD (2010) Extracellular DNA traps promote thrombosis. Proc Natl Acad Sci USA 107:15880-15885.

Fuchs TA, Brill A, and Wagner DD (2012) Neutrophil extracellular trap (NET) impact on deep vein thrombosis. Arterioscler Thromb Vasc Biol 32:1777-1783.

Giraldo E, Martin-Cordero L, Hinchado MD, Garcia JJ, and Ortega E (2010) Role of phosphatidylinositol-3-kinase (PI3K), extracellular signal-regulated kinase (ERK) and nuclear transcription factor kappa beta (NF-k beta) on neutrophil phagocytic process of Candida albicans. Mol Cell Biochem 333:115-120.

Gupta AK, Hasler P, Holzgreve W, Gebhardt S, and Hahn S (2005) Induction of neutrophil extracellular DNA lattices by placental microparticles and IL-8 and their presence in preeclampsia. Hum Immunol 66:1146-1154.

Hakkim A, Fuchs TA, Martinez NE, Hess S, Prinz H, Zychlinsky A, and Waldmann H (2011) Activation of the Raf-MEK-ERK pathway is required for neutrophil extracellular trap formation. Nat Chem Biol 7:75-77.

Hakkim A, Fürnrohr BG, Amann K, Laube B, Abed UA, Brinkmann V, Herrmann M, Voll RE, and Zychlinsky A (2010) Impairment of neutrophil extracellular trap degradation is associated with lupus nephritis. Proc Natl Acad Sci USA 107: 9813-9818.

Hasday JD, Garrison A, Singh IS, Standiford T, Ellis GS, Rao S, He JR, Rice P, Frank $\mathrm{M}$, and Goldblum SE, et al. (2003) Febrile-range hyperthermia augments pulmonary neutrophil recruitment and amplifies pulmonary oxygen toxicity. Am J Pathol 162:2005-2017.

Jiang Q, Cross AS, Singh IS, Chen TT, Viscardi RM, and Hasday JD (2000) Febrile core temperature is essential for optimal host defense in bacterial peritonitis. Infect Immun 68:1265-1270.

Kanaoka Y, Maekawa A, Penrose JF, Austen KF, and Lam BK (2001) Attenuated zymosan-induced peritoneal vascular permeability and IgE-dependent passive cutaneous anaphylaxis in mice lacking leukotriene C4 synthase. J Biol Chem $\mathbf{2 7 6}$ $22608-22613$.

Kim YS, Kim JS, Kwon JS, Jeong MH, Cho JG, Park JC, Kang JC, and Ahn Y (2010) BAY 11-7082, a nuclear factor- $\kappa$ B inhibitor, reduces inflammation and apoptosis in a rat cardiac ischemia-reperfusion injury model. Int Heart $J$ 51:348-353.

Kumar A, Negi G, and Sharma SS (2012) Suppression of NF- $\kappa$ B and NF- $\kappa$ B regulated oxidative stress and neuroinflammation by BAY 11-7082 ( $\mathrm{I} \kappa \mathrm{B}$ phosphorylation inhibitor) in experimental diabetic neuropathy. Biochimie 94:1158-1165.

Landoni VI, Chiarella P, Martire-Greco D, Schierloh P, van-Rooijen N, Rearte B, Palermo MS, Isturiz MA, and Fernández GC (2012) Tolerance to lipopolysaccharide promotes an enhanced neutrophil extracellular traps formation leading to a more efficient bacterial clearance in mice. Clin Exp Immunol 168:153-163.

Manzenreiter R, Kienberger F, Marcos V, Schilcher K, Krautgartner WD, Obermayer A, Huml M, Stoiber W, Hector A, and Griese M, et al. (2012) Ultrastructural characterization of cystic fibrosis sputum using atomic force and scanning electron microscopy. J Cyst Fibros 11:84-92

McDonald PP, Bald A, and Cassatella MA (1997) Activation of the NF-kappaB pathway by inflammatory stimuli in human neutrophils. Blood 89:3421-3433.

Mucke HA (2012) Iguratimod: a new disease-modifying antirheumatic drug. Drugs Today (Barc) 48:577-586.

Nathan C (2006) Neutrophils and immunity: challenges and opportunities. Nat Rev Immunol 6:173-182.

Negrotto S, Malaver E, Alvarez ME, Pacienza N, D’Atri LP, Pozner RG, Gómez RM, and Schattner M (2006) Aspirin and salicylate suppress polymorphonuclear apoptosis delay mediated by proinflammatory stimuli. J Pharmacol Exp Ther 319:972-979.

Office of Laboratory Animal Welfare (1985) Public Health Service Policy on Humane Care and Use of Laboratory Animals. Office of Extramural Research, US Department of Health and Human Services, Bethesda, MD

Pierce JW, Schoenleber R, Jesmok G, Best J, Moore SA, Collins T, and Gerritsen ME (1997) Novel inhibitors of cytokine-induced $I_{\kappa} B \alpha$ phosphorylation and endothelial cell adhesion molecule expression show anti-inflammatory effects in vivo. $J$ Biol Chem 272:21096-21103.

Prescott D and McKay DM (2011) Aspirin-triggered lipoxin enhances macrophage phagocytosis of bacteria while inhibiting inflammatory cytokine production. $A m J$ Physiol Gastrointest Liver Physiol 301:G487-G497.

Remijsen Q, Kuijpers TW, Wirawan E, Lippens S, Vandenabeele P, and Vanden Berghe T (2011) Dying for a cause: NETosis, mechanisms behind an antimicrobial cell death modality. Cell Death Differ 18:581-588.

Scannell M and Maderna P (2006) Lipoxins and annexin-1: resolution of inflammation and regulation of phagocytosis of apoptotic cells. ScientificWorldJournal 6:1555-1573

Segal AW (2005) How neutrophils kill microbes. Annu Rev Immunol 23:197-223.

Serhan CN, Takano T, Chiang N, Gronert K, and Clish CB (2000) Formation of endogenous "antiinflammatory" lipid mediators by transcellular biosynthesis. Lipoxins and aspirin-triggered lipoxins inhibit neutrophil recruitment and vascular permeability. Am J Respir Crit Care Med 161:S95-S101.

Spagnuolo PJ and Ellner JJ (1979) Salicylate blockade of granulocyte adherence and the inflammatory response to experimental peritonitis. Blood 53:1018-1022

Swinney DC, Xu YZ, Scarafia LE, Lee I, Mak AY, Gan QF, Ramesha CS, Mulkins MA, Dunn J, and So OY, et al. (2002) A small molecule ubiquitination inhibitor blocks NF-kappa B-dependent cytokine expression in cells and rats. J Biol Chem 277:23573-23581.

Trevani AS, Andonegui G, Giordano M, López DH, Gamberale R, Minucci F, and Geffner JR (1999) Extracellular acidification induces human neutrophil activation. J Immunol 162:4849-4857.

Tulapurkar ME, Hasday JD, and Singh IS (2011) Prolonged exposure to hyperthermic stress augments neutrophil recruitment to lung during the post-exposure recovery period. Int $J$ Hyperthermia 27:717-725.

Urban CF, Ermert D, Schmid M, Abu-Abed U, Goosmann C, Nacken W, Brinkmann V, Jungblut PR, and Zychlinsky A (2009) Neutrophil extracellular traps contain calprotectin, a cytosolic protein complex involved in host defense against Candida albicans. PLoS Pathog 5:e1000639.

Urban CF, Reichard U, Brinkmann V, and Zychlinsky A (2006) Neutrophil extracellular traps capture and kill Candida albicans yeast and hyphal forms. Cell Microbiol 8:668-676.

von Brühl ML, Stark K, Steinhart A, Chandraratne S, Konrad I, Lorenz M, Khandoga A, Tirniceriu A, Coletti R, and Köllnberger M, et al. (2012) Monocytes, neutrophils, and platelets cooperate to initiate and propagate venous thrombosis in mice in vivo. J Exp Med 209:819-835.

Yin MJ, Yamamoto Y, and Gaynor RB (1998) The anti-inflammatory agents aspirin and salicylate inhibit the activity of $\mathrm{I}_{\kappa} \mathrm{B}$ kinase- $\beta$. Nature 396:77-80.

Address correspondence to: Dr. Mirta Schattner, Pacheco de Melo 3081 (1425) Buenos Aires, Argentina. E-mail: mschattner@hematologia.anm.edu.ar 